\title{
Evaluating the USABILITY OF AN AUTOMATEd COURSE-SPECIFIC VOCABUlaRY TO Measure Language Proficiency in Oral Presentations
}

\author{
Chirag Variawa and Lydia Wilkinson \\ Department of Mechanical and Industrial Engineering, Engineering Communication Program, University of Toronto \\ variawa@mie.utoronto.ca; lydia.wilkinson@utoronto.ca
}

\begin{abstract}
A study is being conducted to automatically identify and highlight course-specific language used in engineering courses. Specifically, the Term-Frequency Inverse-Document Frequency (TF-IDF) algorithm, from the field of computational linguistics, is used to identify words that are characteristic to exams. This paper analyzes the success of a TF-IDF generated word list to capture discipline-specific language in oral design presentations in a second year Environmental Chemistry course.
\end{abstract}

\section{INTRODUCTION}

This paper examines the use of computer-generated wordlists to identify relevant course-specific vocabulary in an engineering classroom. Principles from the fields of computational linguistics, industrial engineering, and higher education are employed to create a software program that performs statistical calculations on groups of input documents to generate keyword lists. Specifically, the program uses a combination of documents from the same discipline and all of engineering to assign a value, called a Term Frequency-Inverse Document Frequency (TF-IDF) score, to each word in an input document. In the studies so far, the words have been extracted from all electronically available engineering exams at the University of Toronto. These documents are used because they are a standardized artifact of the learning environment, and are usually a summative indicator of the learning objectives in different courses. As a result, the wordlists are specific to each course, even though there may be overlap of certain words with other exams - the program finds keywords in documents, and course-specific vocabulary are considered keywords for engineering exams.

The words from each exam are then tabulated in order of decreasing TF-IDF score. The higher the TF-IDF score, the more likely it is that the word is a keyword. However, this approach does have some critical features that limit its applicability in its current form.

\section{USING TF-IDF IN AN ORAL DESIGN PROJECT}

In this study, TF-IDF was used to measure the effectiveness of student's discipline-specific language in a second year environmental chemistry class. We focused specifically on the formal client meeting, an oral presentation that is an important step in the design project. Mapping the word list against vocabulary in the presentation exposed limits arising from differences in purpose and mode between exams and design projects.

CEEA13; Paper 75.

Montreal, OC; June 17-20, 2013
The relative purpose of exams and design projects affects the type of word forms that are used. Exams aim to test knowledge and as such often use the imperative form to deliver a set of instructions. Students are asked to calculate the alkalinity of..., give the equation for...., or estimate the concentration of....in order to display knowledge. In comparison, design projects, and these oral presentations specifically, often use the past or future tense to describe what has been accomplished or what will be done next, explaining for instance that an estimated total will be collected, or that ratios were calculated. A direct mapping of the wordlist against the presentation does not capture the form of the word or acknowledge shared root words.

The skills tested for and used in exams and design projects - knowledge versus problem solving, investigation and project management - also impact vocabulary. Design projects require students to look for solutions outside the lecture content, and as a result words occur in the project that may not be tested for on the exam. For example, in one group of presentations, gas was the most frequently recurring word list term; but while it would be used on an exam to refer to a gas state, it was used here to refer to gasoline manufacturers, key contributors to pollution on the site being evaluated. Gas was also used as a modifier in gas chromatography, and notably, chromatography was the next most frequent word list term. Both in usage and colocation we again see the importance of interpretation in applying the word list.

The list also does not capture acronyms, which are a key expression of professional competency within this context. A student at the beginning of their presentation may indicate that they are testing for Volatile Organic Compounds, but through the rest of the presentation consistently refer to VOC's. While the full term is only captured once, the repeated acronym has the same basic meaning. Within Chemical Engineering condensing terms is a necessary means of ensuring concision within a discipline where lengthy word chains are the norm. A student's inability to recognize acronyms by graduation would suggest a lack of proficiency in their chosen field.

Given the above limitations, it becomes clear that the current approach of using the method as-is is not able to fully characterize the course-specific vocabulary used on oral presentations. Specifically, the program is not yet capable of discerning between acronyms or shorthand, the demonstration of skills in a design course, and differences in word form and usage. Future work will investigate these areas as well as exploring the use of speech-to-text to aid in identifying word usage in oral presentations. 